\title{
Desenvolvimento e controle de qualidade de um gel-creme antiacneico a base do óleo da Copaífera officinalis $L$. (copaíba)
}

\author{
Development and quality control of an anti-acne gel-cream obtained from the \\ essential oil of Copaífera officinalis L. (copaíba)
}

\section{Desarrollo y control de calidad de un gel-crema antiacneico a base del aceite de la Copaífera officinalis L. (copaíba)}

Fábio Vinícius Ferreira Silva ${ }^{1 *}$, Mateus Cavalcante Santos ${ }^{1}$, Luanna Duarte Benvindo Neiva1, Maria Aparecida Coelho Oliveira ${ }^{1}$, Bianca de Sousa Leal1', Francisco Assis dos Santos Moreira1', Patrícia Nunes dos Santos ${ }^{1}$, Gisele Lopes Cavalcante ${ }^{2}$, Jordanna di Paula dos Santos Sousa ${ }^{1}$, Manoel Pinheiro Lúcio Neto ${ }^{3}$.

\section{RESUMO}

Objetivo: Desenvolver um gel-creme antiacneico a base do óleo da Copaífera officinalis $L$. (copaíba) e realizar o controle de qualidade do mesmo. Métodos: Foi desenvolvida uma formulação, e incorporou-se o óleo da planta na mesma, verificou-se a compatibilidade entre ambos. Realizou-se os ensaios organolépticos (aspecto, cor e odor), físico-químicos ( $\mathrm{pH}$, densidade e espalhabilidade) e microbiológicos, nos tempos 0 e 30 dias após a produção, e também os testes de centrifugação e estresse de temperatura visando a previsão de possíveis instabilidades. Resultados: No teste de incorporação, a melhor concentração foi 3,5\%, com ausência de alterações. O teste de centrifugação, não apresentou separação de fases e precipitações, assim como o de estresse térmico, onde o produto foi submetido à elevadas condições de temperatura. O gel-creme apresentou-se na cor levemente verde, aspecto gelificado, odor característico, $\mathrm{pH}$, dentro dos padrões de segurança $(5,0-5,5)$, densidade $0,97420 \mathrm{~g} / \mathrm{cm}^{3}-0.99820 \mathrm{~g} / \mathrm{cm}^{3}$, boa espalhabilidade, esta aumentou à medida em que o peso foi adicionado. Embora a amostra tenha apresentado crescimento microbiano e fungicida, o número de Unidades Formadoras de Colônias encontra-se abaixo do limite máximo. Conclusão: Por conseguinte o gel-creme apresenta um elevado perfil cosmetológico, com ausência de instabilidades, adequado aos padrões de segurança e eficácia especificados.

Palavras-chave: Copaífera officinalis L, Antiacneico, Gel-creme, Controle de qualidade.

\begin{abstract}
Objective: To develop an antiacne gel-cream based on Copaífera officinalis $L$ oil. (copaíba) and to carry out a quality control of the same. Methods: A formulation was developed and the oil extracted from the plant was incorporated in the formulation. The compatibility between the formulation and oil was verified. The organoleptic tests (appearance, color and odor), physical-chemical ( $\mathrm{pH}$, density and spreadability) and microbiological tests were carried out at 0 and 30 days after production, as well as centrifugation and temperature stress tests in order to verify possible instabilities. Results: In the incorporation test, the best concentration was $3.5 \%$, with no alterations. The centrifugation test did not show phase separation and precipitation, as well as thermal stress test, in which the product was subjected to high temperature conditions.
\end{abstract}

${ }^{1}$ Centro Universitário Santo Agostinho - UNIFSA, Teresina-PI.

2Universidade Federal do Piauí - UFPI, Teresina-PI.

3Universidade Luterana do Brasil - ULBRA, Teresina-PI. *E-mail: viniciussaferreira@outlook.com 
The gel-cream exhibited a slightly green color, gel-like appearance, characteristic odor, $\mathrm{pH}$, within safety standards (5.0-5.5), density $0.977420 \mathrm{~g} / \mathrm{cm}^{3}-0.99820 \mathrm{~g} / \mathrm{cm}^{3}$ and good spreadability, this latter increased as the weight was increased. Although the sample showed microbial and fungicidal growth, the number of Colony Forming Units is below the maximum limit. Conclusion: Therefore, the gel-cream displays a high cosmetic profile, with no instabilities, and it is considered adequate according to the specified safety and efficacy standards.

Key words: Copaífera officinalis L., Anti-acne, Gel-cream, Quality control.

\section{RESUMEN}

Objetivo: Desarrollar un gel-crema antiacneico a base del aceite de la Copaífera officinalis L. (copaíba) y realizar el control de calidad del mismo. Métodos: Se desarrolló una formulación, e incorporó el aceite de la planta en la misma, se verificó la compatibilidad entre ambos. Se realizaron los ensayos organolépticos (aspecto, color y olor), físico-químicos ( $\mathrm{pH}$, densidad y esparcimiento) y microbiológicos, en los tiempos 0 y 30 días después de la producción, y también las pruebas de centrifugación y estrés de temperatura visando la previsión de posibles inestabilidades. Resultados: En la prueba de incorporación, la mejor concentración fue 3,5\%, con ausencia de alteraciones. La prueba de centrifugación, no presentó separación de fases y precipitaciones, así como el de estrés térmico, donde el producto fue sometido a altas condiciones de temperatura. El gel-crema se presentó en el color ligeramente verde, aspecto gelificado, olor característico, $\mathrm{pH}$, dentro de los patrones de seguridad $(5,0-5,5)$, densidad $0,97420 \mathrm{~g} / \mathrm{cm}^{3}-0.99820 \mathrm{~g} / \mathrm{cm}^{3}$, buena dispersión, esta aumentó a medida que el peso se añadió. Aunque la muestra presentó crecimiento microbiano y fungicida, el número de Unidades formadoras de colonias se encuentra por debajo del límite máximo. Conclusión: Por lo tanto el gel-crema presenta un alto perfil cosmetológico, con ausencia de inestabilidades, adecuado a los estándares de seguridad y eficacia especificados.

Palabras clave: Copaífera officinalis L, Antiacneico, Gel-crema, Control de calidad.

\section{INTRODUÇÃO}

$\mathrm{Na}$ atualidade, à medida que os consumidores buscam por produtos com qualidades reconhecidas, que atendam a demanda dos padrões de beleza, uma tendência denominada "onda verde" tem se destacado no mercado cosmético e de higiene pessoal (BARBIZAN F, et al., 2013).

Neste cenário, em que a indústria cosmética apresenta um desenvolvimento favorável, a utilização de insumos ativos de origem vegetal, representa uma atitude inovadora, onde materiais sintéticos são substituídos por naturais, e apresenta fundamental relevância no desenvolvimento de novas formulações, e isso deve-se ao fato da vasta extensão fitoecológica e diversidade de biomas existentes no território brasileiro, ademais constitui uma atitude sustentável (ISAAC GEA, 2016).

Deste modo, sendo o Brasil um país com uma extensa biodiversidade, onde a mesma é considerada uma das maiores do mundo, várias plantas são utilizadas como insumo no desenvolvimento de produtos cosméticos, dentre estas destaca-se a Copaífera officinalis $L$., pertencente ao gênero Copaífera, é caracterizada como a principal espécie de copaíba. Dentre as várias atividades terapêuticas apresentadas pelo óleo resina da Copaifera officinalis $L$. (copaíba), é válido mencionar a sua relevante atuação como antiinflamatório, antirreumático, anticancerígeno, antimicrobiana e, principalmente, cicatrizante de úlceras bem como feridas em geral. A inibição do edema, o aumento de tecido de granulação, assim como da permeabilidade vascular, resulta do mecanismo de ação anti-inflamatória. Em virtude de o óleo desta planta ser um ótimo anti-inflamatório e cicatrizante, por apresentar baixa toxicidade, torna-se bastante utilizado no tratamento de uma gama de patologias, assim como na indústria de cosméticos, podendo ser adequado ao desenvolvimento de várias formulações (FRANCISCO SG, 2005). 
Diante disso, evidenciando-se as ações antimicrobiana e anti-inflamatória apresentadas pelo óleo da copaíba, é importante ressaltar que este composto apresenta-se como um importante antiacneico, tendo em vista que a acne é uma patologia que caracteriza-se por possuir um mecanismo em que as glândulas sebáceas quando estimuladas por andrógenos, provocam uma elevação da unidade pilossebácea (HASSUM KM, 2000; PIMENTEL AS, 2008).

Geralmente acomete regiões do corpo que incluem a face, região anterior e posterior do tórax, é definida como uma condição inflamatória crônica dos folículos pilossebáceos, e estimativas mostram que $80 \%$ dos adolescentes no seu ciclo vital, já manifestaram sintomatologia desta doença (ALGAYER N, 2014).

O óleo de copaíba possui importantes características, entre elas: atua modificando as secreções e acelerando o processo de cicatrização, e isso deve-se ao restabelecimento das funções das membranas mucosas, alguns estudos sugerem que a presença de sesquiterpenos no óleo é a responsável pela ação antiinflamatória, o que faz dessa substância uma excelente alternativa para uso em formulações cosméticas antiacneicas (MOREIRA MEC, et al, 2008).

Uma ótima opção de cosméticos para aplicação cutânea, ou em mucosas, são as preparações tópicas semissólidas, onde estão incluídos os géis- creme, uma vez que apresentam ação local ou penetração percutânea de medicamentos, além do mais possuem ação emoliente ou protetora (BRASIL, 2010; ANTONIO MECO, 2007).

O crescimento do uso de ativos de origem natural em formulações cosméticas representa um episódio imprescindível no mercado, visto que diante deste panorama econômico favorável, os cosméticos sustentáveis são de fundamental relevância, uma vez que são inovadores e pioneiros (ISAAC GEA, 2016).

Por apresentarem atividades cicatrizantes ou preventivas de sinais do envelhecimento, esses ingredientes naturais têm sido muito utilizados, quando se diz respeito aos cuidados com a pele, e isso justifica-se pelo fato de representarem uma alternativa para os consumidores que após se depararem com os efeitos adversos de outros produtos enfrentaram muitas frustrações, e diante disso anseiam por produtos seguros e ecológicos (SOUZA IDS e TAKAHASHI VP, 2015).

Todavia, considerando-se a constante busca por novas alternativas cosméticas, fato que intensifica a competição no mercado, uma excelente opção, é a utilização de gel-creme, que consiste em uma forma cosmética classificada com emulsão constituída por uma elevada porcentagem de fase aquosa e baixa quantidade de conteúdo oleoso (CHORILLI M, et al., 2006).

Em meio a um cenário onde o consumidor mostra-se cada vez mais crítico e exigente, quanto à escolha dos produtos cosméticos, é válido ressaltar que quando esses produtos de origem natural apresentam qualidade cientificamente comprovada, o interesse por parte dos clientes é aumentado, diante do exposto é relevante destacar a necessidade da realização do controle de qualidade destes produtos, visando garantir a segurança e eficácia dos mesmos (FERRARI M, et al., 2007).

Diante disso, o presente trabalho objetivou desenvolver um gel-creme antiacneico a base de óleo da Copaífera officinalis $L$. (copaíba) e realizar o controle de qualidade do mesmo, tendo em vista que o desenvolvimento deste produto é de grande relevância para o mercado cosmético, uma vez que apresenta formulação desenvolvida dentro dos padrões de qualidade, com atividade antiacneica e promove a hidratação da pele.

Considerando a fitoquímica desta planta e os estudos de literatura científica é válido afirmar que a mesma é provida de uma gama de constituintes bioativos, onde podem ser destacados com propriedade, os sesquiterpenos, que são os responsáveis pela atividade anti-inflamatória, atuando deste modo na inibição do processo inflamatório cutâneo causado pela acne.

Ademais, o delineamento de formulações cosméticas com a utilização de princípios ativos de origem vegetal constitui uma questão também de caráter ecológico, e corrobora assim para uma melhor exploração da biodiversidade brasileira. 


\section{MÉTODOS}

\section{Escolha e Aquisição da Matéria-Prima}

As matérias-primas foram adquiridas em setembro de 2018 no Serviço Escola de Farmácia do Centro Universitário Santo Agostinho - UNIFSA (Avenida Barão de Gurguéia, n 2642, Bairro São Pedro de Teresina$\mathrm{PI}$ ), que foram analisadas através de estudo de pré-formulação, para predizer a compatibilidade da formulação com o óleo da Copaifera officinalis L.(copaíba), obtido na Farmácia Galeno (Rua São Pedro, no 2235, Bairro Centro (Sul), Teresina - PI).

\section{Preparo da formulação base}

A formulação base do gel-creme antiacneico foi preparada no Laboratório de Ciências Farmacêuticas do UNIFSA, seus componentes, funções e respectivas concentrações se encontram descritas no (Quadro 1).

Inicialmente, pesou-se a cera auto emulsionante, o carbopol e o metilparabeno, separadamente. Posteriormente preparou-se em recipientes separados, a fase aquosa e a fase oleosa, colocou-se os componentes de cada fase separadamente em um béquer de $100 \mathrm{~mL}$, prosseguiu-se com a dissolução dos mesmos. Em seguida aqueceu-se separadamente a fase oleosa a $80^{\circ} \mathrm{C}$ e a fase aquosa a $85^{\circ} \mathrm{C}$. Logo após o aquecimento, verteu-se a fase aquosa sobre a oleosa, sob agitação até o momento em que se atingiu a temperatura ambiente. Por fim, adicionou-se a vitamina $\mathrm{C}$, homogeneizou-se e consecutivamente adicionouse a trietanolamina lentamente, sob agitação até obter a gelificação, acertou-se o pH, adicionou-se o corante e a essência e completou-se com água destilada q.s.p, 100,0 mL (BRASIL, 2008). As formulações preparadas seguiram-se as boas práticas de manipulação e controle, preconizados pela Resolução da Diretoria Colegiada (RDC) 67/2007 da Agência Nacional de Vigilância Sanitária (ANVISA). Logo em seguida após a sua preparação foram aplicados testes de estabilidade. Tendo em vista que o objetivo do trabalho foi 0 desenvolvimento do gel-creme e a realização do controle de qualidade do mesmo, não se realizou nenhum teste de verificação da eficácia do produto no controle da acne, assim como da vida de prateleira.

\section{Incorporação do óleo de Copaífera officinalis L. (copaíba)}

Primeiramente, dividiu-se a formulação base em três recipientes contendo concentrações iguais da mesma em cada um, em seguida, realizou-se a incorporação do óleo da Copaífera officinalis $L$. (copaíba) na formulação, nas seguintes concentrações: $1,5 \%$, no recipiente (01); 3,5\% no recipiente (02) e 4,0\%, no recipiente (03). Posteriormente observou-se qual a concentração que melhor incorporou-se à formulação e aos poucos, por meio de movimentos circulares, verificou-se visualmente possíveis alterações na viscosidade e coloração, ou a ocorrência de separação de fases ou sedimentação para a escolha da melhor concentração, que foi utilizada para posterior realização dos demais testes.

\section{Teste de centrifugação}

A centrifugação produz estresse na amostra, simulando um aumento na força de gravidade, aumentando a mobilidade das partículas e antecipando possíveis instabilidades. Estas poderão ser observadas na forma de precipitação, separação de fases, formação de sedimento compacto e coalescência, entre outras. Para a realização do teste, empregou-se $5 \mathrm{~g}$ de amostra, em triplicata e centrifugou-se a 300rpm por $30 \mathrm{~min}$ em temperatura ambiente. Em seguida, procedeu-se à avaliação visual para análise macroscópica, para a verificação da ocorrência de instabilidade física, como separação de fases ou cremeação (BRASIL, 2008).

\section{Teste de Estresse de Temperatura}

A amostra foi submetida a condições extremas de temperaturas com o intuito de antecipar possíveis instabilidades, no qual se pesou aproximadamente $5 \mathrm{~g}$ da amostra, onde foram submetidas a aquecimento em banho-maria nas faixas $40,50,60$ e $70^{\circ} \mathrm{C}$, mantendo-se por 30 minutos em cada temperatura, posteriormente prossegue-se com a análise visual para a verificação de possíveis instabilidades (LIMA VC, et al., 2008; VELASCO MRV, et al., 2008).

\section{Avaliação da estabilidade da formulação}




\section{Estabilidade preliminar- condições ambientais de temperatura}

A amostra foi submetida a condições ambientais de temperaturas com 0 intuito de simular 0 armazenamento doméstico, em um período de 0 a 30 dias, onde foram avaliadas nos tempos 0 e 30 dias após a produção as propriedades organolépticas e físico-químicas (BRASIL, 2004).

\section{Ensaios Organolépticos}

Esses ensaios fornecem parâmetros que permite avaliar, de imediato, o estado da amostra em estudo. Estes parâmetros foram analisados nos tempos 0 e após 30 dias da produção e acondicionamento em temperatura ambiente com o objetivo de verificar alterações, possibilitando o reconhecimento primário do produto, utilizando os dados obtidos no tempo 0 (ANVISA, 2004).

\section{Aspecto}

Observou-se visualmente se a amostra em estudo manteve as mesmas características "macroscópicas" dos dados obtidos no tempo 0 ou se ocorreram alterações do tipo separação de fases, precipitação, turvação, etc. (ANVISA, 2017; ANVISA, 2004).

\section{Cor}

Comparou-se visualmente a cor da amostra com os dados obtidos no tempo 0 , sob luz "branca" natural (ANVISA, 2017; ANVISA, 2004).

\section{Odor}

A amostra teve o seu odor inicial e final comparado diretamente através do olfato (ANVISA, 2017; ANVISA, 2004).

\section{Ensaios Físico-Químicos}

São operações técnicas que determinam uma ou mais características de um produto de acordo com um procedimento especificado foram realizadas no tempo 0 e após 30 dias da produção (ANVISA, 2004).

$\mathrm{pH}$

Preparou-se a amostra diluindo-se $1 \mathrm{~g}$ de formulação em $10 \mathrm{~mL}$ de água destilada. Em seguida essa solução foi levada ao pHmetro, de forma que se obteve o pH característico da formulação (ANVISA, 2004).

\section{Densidade Aparente}

Pesou-se uma quantidade da amostra no qual foi introduzida em uma proveta para se medir o volume e os valores obtidos foram introduzidos na fórmula da densidade aparente.

$$
\text { Cálculo: } D=\frac{m}{v}
$$

Onde: $\mathrm{D}=$ densidade aparente $\mathrm{em} \mathrm{g} / \mathrm{cm}^{3}, \mathrm{~m}=$ massa da amostra em $\mathrm{g}, \mathrm{v}=$ volume final $\mathrm{em}^{\mathrm{cm}}{ }^{3}$ (ANVISA, 2017; ANVISA, 2004).

\section{Teste de Espalhabilidade}

Realizou-se a determinação da espalhabilidade da amostra pesando $0,3 \mathrm{~g}$ da amostra e colocando entre duas placas de vidro, dispostas sobre um papel milimetrado.

A cada 3 minutos adicionou-se pesos de 50, 150, 250 e 350g sobre a placa superior e realizou-se a leitura em diâmetros da espalhabilidade. Os valores obtidos foram utilizados para calcular a espalhabilidade (BRASIL, 2008).

Cálculo da espalhabilidade:

$$
E i=d^{2} \times \frac{\pi}{4}
$$

Onde: $\mathrm{Ei}=$ espalhabilidade da amostra para um determinado peso i $(\mathrm{mm} 2)$; d2 = diâmetro médio $(\mathrm{mm})$. 


\section{Ensaio Microbiológico}

Realizou-se nos tempos 0 e após 30 dias da produção, uma diluição de 1:10, transferindo $10 \mathrm{~mL}$ de amostra para o erlenmeyer de $100 \mathrm{~mL}$ completando até volume final com solução de Caldo Caseína-soja e homogeneizando; e uma diluição de 1: 100 seguindo o mesmo processo da anterior. Posteriormente, aplicouse $1 \mathrm{ml}$ de cada diluição em duas placas de Ágar Caseína soja e duas de Ágar Sabouraud, sendo uma para cultura de bactérias e outra para fungos; incubou-se as placas de Ágar Caseína-soja em estufa a $40 \pm 5^{\circ} \mathrm{C}$ por 5 dias, e de Ágar Sabouraud a $40 \pm 3^{\circ} \mathrm{C}$ durante 7 dias e realizou-se uma contagem visual do número de colônias em cada placa (BRASIL, 2010).

\section{Análises Estatísticas}

Os dados quantitativos obtidos durante o estudo foram arranjados e analisados com recursos estatísticos de suporte da análise expressados por intermédio de gráficos e tabelas utilizando os softwares Microsoft Office Excel e Word.

\section{RESULTADOS E DISCUSSÃO}

A princípio, realizou-se mediante estudos de literatura científica e bancada laboratorial o delineamento de uma formulação que fosse adequada ao desenvolvimento do gel-creme antiacneico. Logo após, iniciou-se o processo de manipulação do produto, obtendo-se assim um cosmético com atividade antiacneica, dentre as quais podem ser destacadas as características visualmente perceptíveis (Quadro 1).

Quadro 1 - Componentes, Funções e Concentrações (em \%) da Formulação Teste.

\begin{tabular}{|c|c|c|c|}
\hline FASES & COMPONENTE & FUNÇÃO & CONCENTRAÇÃO \% \\
\hline OLEOSA & $\begin{array}{l}\text { Cera auto emulsionante- } \\
\text { Polavax } \\
\text { Vaselina líquida }\end{array}$ & $\begin{array}{l}\text { Emulsificante } \\
\text { Constituinte da base } \\
\text { (fase oleosa) }\end{array}$ & $\begin{array}{l}6 \% \\
2 \%\end{array}$ \\
\hline AQUOSA & $\begin{array}{l}\text { Carbopol } \\
\text { Metilparabeno } \\
\text { Propilenoglicol } \\
\text { Trietanolamina (ou NaOH 10\% } \\
\text { ou AMP-95) } \\
\text { Corante } \\
\text { Vitamina C } \\
\text { Essência } \\
\text { Água destilada q.s. p }\end{array}$ & $\begin{array}{l}\text { Espessante/gelificante } \\
\text { Conservante } \\
\text { Umectante e } \\
\text { Co-solvente } \\
\text { Corretivo de Ph } \\
\text { Coloração } \\
\text { Antioxidante } \\
\text { Aromatizante } \\
\text { Veículo }\end{array}$ & $\begin{array}{c}0,4 \% \\
0,15 \% \\
2 \% \\
\text { q.s. } \\
\text { q.s. } \\
1 \% \\
\text { q.s. } \\
100,0 \mathrm{~mL}\end{array}$ \\
\hline
\end{tabular}

Legenda: q.s. = quantidade suficiente.

Fonte: Dados da pesquisa, 2018.

Os géis-creme constituem uma excelente forma farmacêutica e/ou cosmética, para o desenvolvimento de cosméticos, tendo em vista que estes são absorvidos rapidamente, e apresentam facilidade de incorporação dos ativos (LEONARDI GR, 2008; SILVA PM e SILVA MAE, 2009). Assim, uma prática bastante difundida é a incorporação dos extratos vegetais em bases para fins cosméticos, deste modo, a escolha adequada da base à qual os princípios ativos de uso tópico serão incorporados é de fundamental importância, pois corrobora para garantir que os efeitos farmacodinâmicos esperados sejam alcançados, e os uma melhor absorção dos princípios ativos através da manutenção da estabilidade (BUHLER FV e FERREIRA JRN, 2008).

Após o teste de incorporação do óleo de Copaífera officinalis L. (copaíba) na formulação base, observouse que a concentração que melhor incorporou-se à formulação foi a intermediária de $3,5 \%$ onde por meio de movimentos circulares, observou-se visualmente uma boa incorporação do óleo na base do gel-creme, com ausência de alterações no aspecto, bem como na viscosidade e coloração, não ocorrência de separação de fases ou sedimentação, não houve quebra da consistência sendo esta escolhida como a melhor concentração, que foi utilizada para posterior realização dos demais testes (Figura 1). 
Figura 1 - Gel-creme antiacneico com óleo de copaíba.

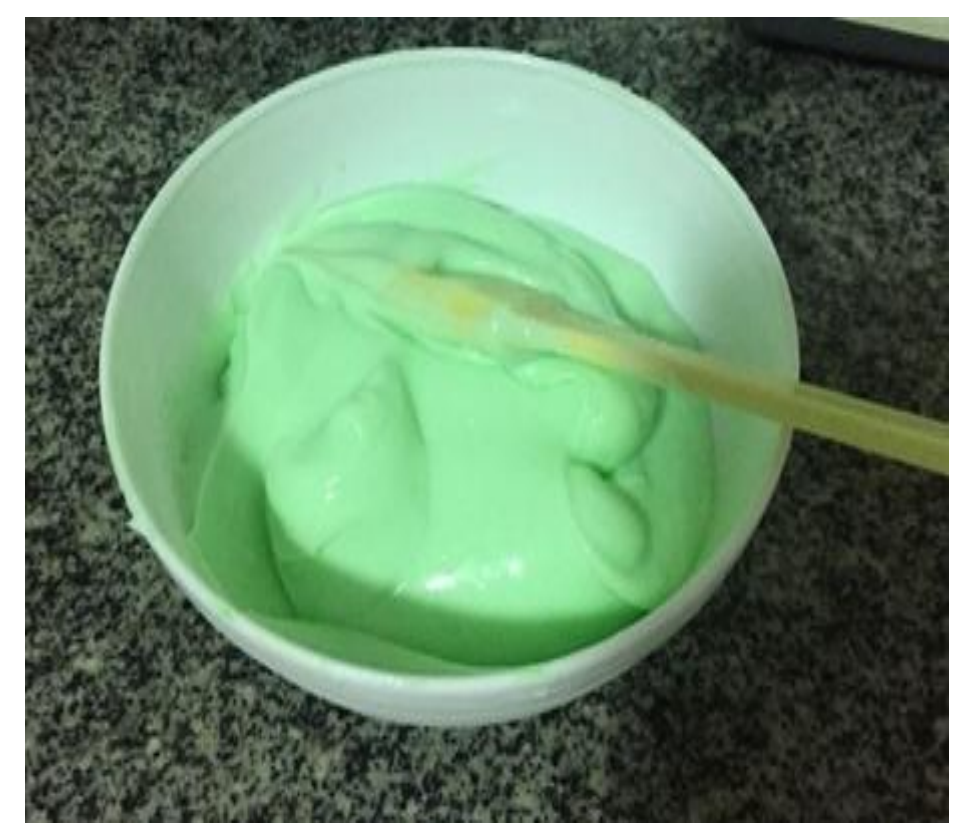

Fonte: Dados da pesquisa, 2018.

Em conformidade com o Guia de Controle de Qualidade de Produtos Cosméticos da ANVISA (2008), o teste de centrifugação consiste em uma atuação da força da gravidade sobre os produtos, fazendo com que em seu interior haja uma movimentação das partículas. A centrifugação produz estresse na amostra, simulando um aumento na força de gravidade, aumentando a mobilidade das partículas e antecipando possíveis instabilidades. Estas poderão ser observadas na forma de precipitação, separação de fases, formação de sedimento compacto (caking) e coalescência, entre outras.

As amostras são centrifugadas em temperatura, tempo e velocidade padronizados. Com a realização desse teste, objetivou-se em um curto espaço de tempo, avaliar possíveis instabilidades físicas e químicas que podem atingir as formulações. Após a realização do mesmo, os resultados obtidos não demonstram nenhum sinal de separação de fase e precipitações como floculação ou coalescência, cremeação, dentre outras, não havendo assim indícios de instabilidade, a mesma apresentou-se estável e não houve necessidade de reformulação, onde foi submetido posteriormente aos testes de estabilidade (ANDRADE FF, 2008) (Tabela 1).

Tabela 1 - Características organolépticas, $\mathrm{pH}$, densidade e teste de centrifugação das formulações nos tempos 0 e 30 dias após a produção.

\begin{tabular}{c|c|c}
\hline Análise & Tempo (em dias) & Formulação \\
\hline Aspecto & 0 & Gelificado \\
& 30 & - \\
\hline Cor & 0 & Levemente verde \\
\hline Odor & 30 & - \\
\hline pH & 0 & Característico \\
\hline Densidade & 30 & - \\
\hline (g/cm $^{3}$ & 0 & 5,0 \\
\hline Centrifugação & 0 & 5,5 \\
\hline
\end{tabular}

Fonte: Dados da pesquisa, 2018. 
A instabilidade de formulações cosméticas pode ser detectada em alguns casos através de alterações físicas no aspecto, cor e odor das formulações (ALLEN JÚNIOR LV, 2002). Com relação às características organolépticas da formulação, a mesma não apresentou alterações quanto a coloração, odor e aspecto. $O$ gel-creme apresentou-se na cor levemente verde, aspecto gelificado, odor característico, mantendo-se estável macroscopicamente (Tabela 1).

Conforme DECCACHE DS (2006), fatores como: a compatibilidade dos componentes da formulação, eficácia, e segurança de uso, possuem relação com a determinação do $\mathrm{pH}$, sendo estes nos estudos de estabilidade um importante parâmetro de monitoração a ser avaliado. Ainda assim, a qualidade dos produtos pode ser comprometida pela presença de reações químicas que resultam da alteração do potencial hidrogeniônico (pH) (ANDRADE FF, 2008).

$\mathrm{O} \mathrm{pH}$, do gel-creme apresentou-se dentro dos padrões de segurança $(5,0-5,5)$, conforme disposto, sendo este adequado ao pH cutâneo, o que corrobora para a redução dos riscos de impacto na pele, bem como para a obtenção de um produto mais estável e seguro para uso. O potencial hidrogeniônico $(\mathrm{pH})$, da pele dependendo da região do corpo e idade, possui variações, sendo o pH fisiológico entre 4,6 e 6,0, é considerado como levemente ácido, contribuindo assim para a proteção da superfície cutânea contra a ação de bactérias e fungos.

Ainda assim, as secreções da pele, possuem uma capacidade tamponante apreciável, propriedade muito importante, uma vez que quando os produtos tópicos são utilizados de maneira inadequada, o pH é frequentemente alterado, o que leva a exposição da pele a uma série de agentes agressores, em especial, microrganismos (LEONARDI GR; GASPAR LR; CAMPOS PMBG, 2002).

Portanto, a variação nos valores de $\mathrm{pH}$, do gel-creme podem ser consideradas normais, uma vez que se encontram dentro da faixa de $\mathrm{pH}$, correspondente aos parâmetros ideais de uma formulação cosmética (Tabela 1).

Em conformidade com Rebelo T e Bezerra SV (2001), a emulsão pode ser desestabilizada, e parâmetros como a cor e o odor, podem ser alterados em virtude da presença de ar. Essa incorporação de ar no produto pode ser detectada através da densidade, uma vez que se houver diminuição da densidade, significa que houve incorporação de ar. No entanto, no que se refere à densidade do gel-creme antiacneico $0,97420 \mathrm{~g} / \mathrm{cm}^{3}$ - $0.99820 \mathrm{~g} / \mathrm{cm}^{3}$, pode-se afirmar que não houve incorporação de ar, tendo em vista que esta aumentou no intervalo de tempo de 0 a 30 dias.

De acordo com Guia de Controle de Qualidade de Produtos Cosméticos da ANVISA (2008), o valor da densidade do gel-creme ficou dentro do escopo estipulado $(0,95-1,05 \mathrm{~g} / \mathrm{cm} 3)$ ao produto, média encontrada em outros estudos de desenvolvimento de géis-creme, o mesmo não apresenta interferência, conforme preconizado pela especificação de qualidade. A amostra foi submetida a condições de estresse térmico para avaliação da estabilidade preliminar, tendo em vista que esse teste visa acelerar processos de instabilidade, como a degradação de componentes da formulação.

Observou que a amostra se manteve estável após o teste, com a fluidez aumentada em virtude do aquecimento, um odor característico mais forte, devido a evaporação da essência. Entretanto não houve separação de fases, mesmo submetida à elevadas condições de temperatura (ANVISA, 2010; LIMA VC, et al., 2008; VELASCO MRV, et al., 2008) (Tabela 1).

A capacidade que uma formulação tem de se espalhar, quando é submetida a uma determinada força, é determinada pelo teste de espalhabilidade, onde procura-se reproduzir as condições de esforço necessárias para aplicação na pele, e está relacionado com a viscosidade (SPELLMEIER F, 2005). Do ponto de vista sensorial, assim como de eficácia, é muito importante as características de espalhamento de uma emulsão sobre a pele (ISAAC VLB, et al., 2008).

De acordo com o Gráfico 1, a espalhabilidade do gel-creme aumentou à medida em que o peso foi adicionado, apresentando assim uma boa espalhabilidade, com capacidade de espalhar-se e abranger o local de ação. 
Gráfico 1 - Espalhabilidade da formulação em função do peso adicionado no tempo 30 dias após a produção.

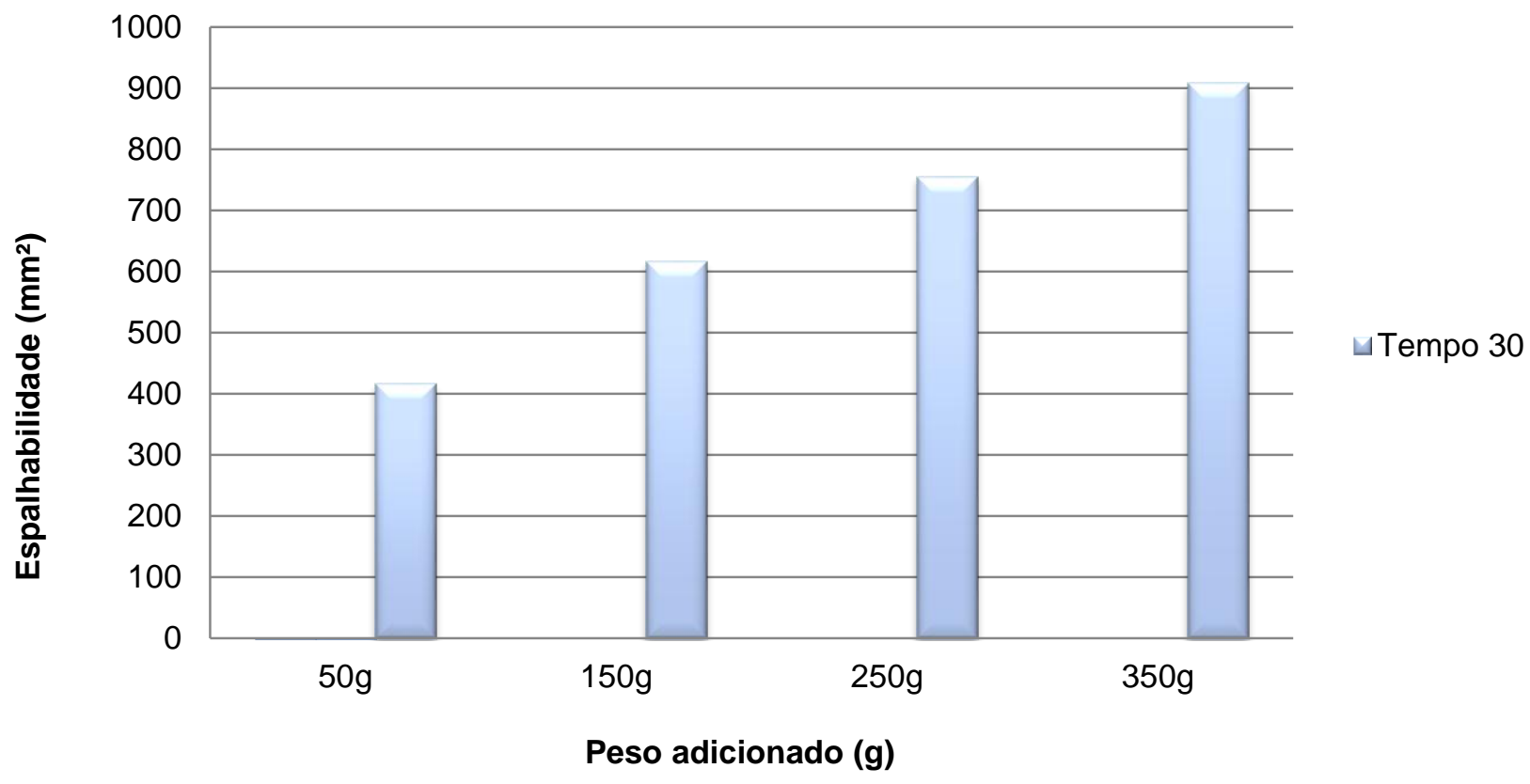

Fonte: Dados da pesquisa, 2018.

A contaminação com micro-organismos acomete praticamente todos os produtos cosméticos. Tendo como objetivo imediato, comprovar a ausência de micro-organismos patogênicos e a determinação do número de células microbianas viáveis, o controle de qualidade microbiológico de produtos não estéreis, admite a presença de carga microbiana limitada, em função da utilização do produto (ANDRADE F, et al., 2005).

Diante disso, conforme com os resultados do controle microbiológico, dispostos, ambas as placas específicas para o crescimento de bactérias e de fungos respectivamente, contendo a formulação, analisada nos tempos 0 e 30 dias após a produção não apresentaram crescimento de microrganismos fora dos padrões especificados.

Em conformidade com a Farmacopeia Brasileira (IV edição), o limite máximo permitido para bactérias aeróbias mesófilas é de $10^{3} \mathrm{UFC} / \mathrm{g}$ e estabelece ainda que o limite máximo para fungos é de $10^{2} \mathrm{UFG} / \mathrm{G}$. Portanto, embora a amostra tenha apresentado crescimento microbiano e fungicida, o número de Unidades Formadoras de Colônias encontra-se abaixo do limite máximo de $10^{3} \mathrm{UFC} / \mathrm{g}$ para bactérias e $10^{2} \mathrm{UFC} / \mathrm{g}$ para fungos (Tabela 2).

Tabela 2 - Resultado do Ensaio Microbiológico realizado com a formulação no tempo 0 e após 30 dias da produção.

\begin{tabular}{c|c|c} 
Microrganismo & $\begin{array}{c}\text { Tempo: Imediatamente após a } \\
\text { produção } \\
\text { Resultado (UFC/g) }\end{array}$ & $\begin{array}{c}\text { Tempo: 30 dias após a } \\
\text { produção } \\
\text { Resultado (UFC/g) }\end{array}$ \\
\hline Bactérias & Negativo & $1,5 \times 10^{3}$ \\
\hline Fungos & Negativo & $1,0 \times 10^{2}$ \\
\hline
\end{tabular}

UFC = Unidade Formadora de Colônias

Fonte: Dados da pesquisa, 2018. 


\section{CONCLUSÃO}

Por conseguinte, o gel-creme antiacneico a base do óleo da Copaífera officinalis L. (copaíba) constitui um produto de perfil cosmetológico elevado, uma vez que possui compatibilidade entre o óleo e a formulação base, com ausência de instabilidades, adequado aos padrões físico-químicos, de estabilidade comprovada durante todo o armazenamento, aprovado em todos os testes aplicados, ademais apresenta carga microbiana dentro dos padrões especificados pela legislação, o que o torna eficaz e seguro ao uso, sendo o controle de qualidade, desta maneira, de grande relevância para a promoção da segurança cosmológica.

\section{REFERÊNCIAS}

1. AGENCIA NACIONAL DE VIGILÂNCIA SANITÁRIA. Guia de controle de qualidade de produtos cosméticos. Agencia Nacional de Vigilância Sanitária. 2a edição, revista - Brasília: ANVISA, 2008.

2. ALLGAYER N. Cicatrizes da acne vulgaris- revisão de tratamentos, 2014.

3. ALLEN JÚNIOR, L. V. Estabilidade de medicamentos manipulados. São Paulo: Rx Editora e Publicidade Ltda, 2002.

4. ANDRADE FF. Desenvolvimento e avaliação de cristais líquidos obtidos em emulsões O/A à base de óleo de andiroba e éster fosfórico. Dissertação (Mestrado). Faculdade de Ciências Farmacêuticas - Universidade de São Paulo, Ribeirão Preto, 151p. 2008.

5. ANDRADE F, et al. Análise microbiológica de matérias-primas e formulações farmacêuticas magistrais. Revista Eletrônica de Farmácia, Goiânia, v. 2, n. 2, p. 38-44, nov. 2005.

6. ANTONIO MECO. Permeação cutânea in vitro como ferramenta auxiliar para o estudo de formulações semi-sólidas de cetoconazol para aplicações tópicas. Dissertação (Pós-Graduação em Ciências Farmacêuticas) - Universidade Federal do Paraná, 2007.

7. BARBIZAN F, FERREIRA EC, TESCAROLLO IL. Sabonete em barra produzido com Óleo de oliva (Olea europea L.) como proposta para o desenvolvimento de cosméticos verdes. Biofar Rev. Biol. Farm. v.9, n.1, p1-6, 2013.

8. BRASIL. Ministério da Saúde. ANVISA - AGÊNCIA NACIONAL DE VIGILÂNCIA SANITÁRIA. Farmacopeia Brasileira, 5.ed. v.2. Brasília: ANVISA, 2010.

9. BRASIL. Ministério da Saúde. Agencia Nacional de Vigilância Sanitária. Guia de Controle de Qualidade de Produtos Cosméticos 2. ed. Brasília: 121 p. ANVISA, 2008.

10. BRASIL. Agência Nacional de Vigilância Sanitária (ANVISA). Gerencia Geral de Cosméticos. Guia de guia de estabilidade de produtos cosméticos. Brasília. Brasília, DF, 2004.

11. BUHLER FV, FERREIRA JRN. Desenvolvimento e avaliação da estabilidade de formulações contendo extratos de llex paraguariensis St. Hil. a 5 e 10\%. Rev Perspect. v. 32, p.47-55. 2008.

12. CHORILLI M, UDO MS, CAVALLINI ME, et al. Desenvolvimento e estudos preliminares de estabilidade de formulações fotoprotetoras contendo Granlux GAI-45 TS®. Revista de Ciências Farmacêuticas Básica e Aplicada, v.27, n.3, p. $267-264,2006$.

13. DECCACHE DS. Formulação dermocosmética contendo DMAE glicolato e filtros solares: desenvolvimento de metodologia analítica, estudo de estabilidade e ensaio biometria cutânea. Rio de Janeiro: UFRJ/ Faculdade de Farmácia, 2006.

14. FRANCISCO SG. Uso do óleo de copaíba (Copaifera officinalis L) em inflamação ginecológica. Feminina, v. 33, n. 2, p. 89-93, fev. 2005.

15. FERRARI M, OLIVEIRA MSC, NAKANO AK, et al. Determinação do fator de proteção solar (FPS) in vitro e in vivo de emulsões com óleo de andiroba (Carapa guianensis). Revista Brasileira de Farmacognosia, v. 17, n. 4, p 626-630, 2007.

16. HASSUM KM. Acne etiopatogênia. Anais brasileiros de dermatologia, v.75, n.1,jan/fev.2000

17. ISACC GEA. O desenvolvimento sustentável do setor cosmético e o comportamento do consumidor frente aos cosméticos sustentáveis. São João da Boa Vista, 2016.

18. ISAAC VLB, CEFALI LC, CHIARI BG, et al. Protocolo para ensaios físico-químicos de estabilidade de fitocosméticos. Revista de Ciências Farmacêuticas básica e Aplicada, v. 29, n. 1, p 81-96, 2008.

19. LEONARDI GR. Cosmetologia aplicada. 2. ed. São Paulo: Santa Isabel, 2008.

20. LEONARDI GR, GASPAR LR, CAMPOS PMBG. Estudo da variação do $\mathrm{pH}$ da pele humana exposta à formulação cosmética acrescida ou não das vitaminas A, E ou de ceramida, por metodologia não invasiva. Anais Brasileiros de Dermatologia. Rio de Janeiro, set/out-2002.

21. LIMA GC, et al. Desenvolvimento e avaliação da estabilidade física de emulsões O/A contendo óleo de babaçu (Orbignya olrifera). Rev. Bras. Farm. v.89, n.3, p.239-245, 2008.

22. MOREIRA MEC, et al. Atividade antiinflamatória de carboidrato produzido por fermentação aquosa de grãos de kefir. Quimica nova, v31, n7, São Paulo, 2008.

23. PIMENTEL AS. Peeling, máscara e acne: Seus tipos e passo a passo do tratamento estético. São Paulo: Livraria Médica e Paulista Editora, 2008.

24. REBELLO T, BEZERRA SV. Guia de Produtos Cosméticos. São Paulo: Editora SENAC São Paulo, 2001.

25. SILVA PM, SILVA MA. E. Índice de saponificação de óleo vegetal constituinte de uma emulsão. $12^{\circ}$ Congresso de iniciação científica: 6ª mostra de pesquisa da Pós-Graduação. São Paulo, p. 952-955, 2009.

26. SOUZA IDS, TAKAHASHI VP. Prospecção de ingredientes ativos naturais para os cuidados com a pele: uma análise de patentes. 2015

27. SPELLMEIER F. Estudo comparativo entre dois métodos de produção de bases emulsionadas através da estabilidade acelerada. 2005. 98 f. Monografia (Graduação) - Curso de Farmácia, Centro Universitário UNIVATES, Lajeado, nov. 2005.

28. VELASCO MVR, et al. Desenvolvimento e teste preliminar da estabilidade de formulações cosméticas acrescidas do extrato comercial de Trichilia catinguá Adr. Juss (e) Ptychopetalum olacoides Bentham. Rev. Ciênc. Farm. Básica Apl. v.29, n.2, p.181196, 2008. 